\title{
Fibroblast Growth Factor 23 and aKlotho in Acute Kidney Injury: Current Status in Diagnostic and Therapeutic Applications
}

\author{
Javier A. Neyra ${ }^{a, b, c}$ Ming Chang Hu ${ }^{a, b}$ Orson W. Moe ${ }^{a, b, d}$ \\ ${ }^{a}$ Charles and Jane Pak Center for Mineral Metabolism and Clinical Research, Dallas, TX, USA; ${ }^{b}$ Department of Internal \\ Medicine, University of Texas Southwestern Medical Center, Dallas, TX, USA; 'Department of Medicine, Division of \\ Nephrology, Bone and Mineral Metabolism, University of Kentucky, Lexington, KY, USA; dDepartment of Physiology, \\ University of Texas Southwestern Medical Center, Dallas, TX, USA
}

\section{Keywords}

Acute kidney injury · aKlotho · Biomarker · Chronic kidney disease $\cdot$ FGF23 - Treatment

\begin{abstract}
Fibroblast growth factor (FGF) 23 and aKlotho are circulating mineral regulatory substances that also have a very diverse range of actions. Acute kidney injury (AKI) is a state of high FGF23 and low aKlotho. Clinical association data for FGF23 are strong, but the basic pathobiology of FGF23 in AKI is rather sparse. Conversely, preclinical data supporting a pathogenic role of aKlotho in AKI are strong, but the human data are still being generated. This pair of substances can potentially serve as diagnostic and prognostic biomarkers. FGF23 blockade and aKlotho restoration can have prophylactic and therapeutic utility in AKI. The literature to date is briefly reviewed in this article.

(c) 2020 S. Karger AG, Basel
\end{abstract}

\section{Introduction}

Fibroblast growth factor (FGF) 23 was cloned as a candidate gene of autosomal dominant hypophosphatemic rickets and a differentially expressed gene in tumor-in- duced osteomalacia, which ushered in a major hormone countering phosphate accumulation [1]. Beyond phosphate balance, FGF23 has been shown or postulated to regulate calcium and sodium homeostasis, iron metabolism, erythropoiesis, and inflammation [2].

aKlotho was cloned when serendipitous disruption of its locus led to premature multiorgan failure [3]. The list of cellular actions conferred by aKlotho encompasses anti-oxidation, anti-apoptosis, pro-autophagy, pro-stem cell, and anti-fibrosis and essentially broad-based maintenance of cell health [1].

Chronic kidney disease (CKD) is a state of FGF23 excess and aKlotho deficiency, but their levels and roles in acute kidney injury (AKI) are less well defined. Many of the functions of FGF23 and aKlotho are highly relevant to the pathobiology of AKI. AKI is a systemic syndrome triggered by a sudden loss of kidney function and brings forth significant morbidity, mortality, and burden on

$\begin{aligned} & \text { karger@karger.com } \\ & \text { www.karger.com/nef }\end{aligned}$
Karger ${ }^{\prime /}$

Javier A. Neyra and Ming Chang Hu contributed equally.

Contribution from the AKI and CRRT 2020 Symposium at the 25th International Conference on Advances in Critical Care Nephrology, Manchester Grand Hyatt, San Diego, CA, USA, February 24-27, 2020. This symposium was supported in part by the NIDDK funded University of Alabama at Birmingham-University of California San Diego O'Brien Center for Acute Kidney Injury Research (P30DK079337). 
multiple organ systems $[4,5]$. Even when one evades the acute high mortality, survivors are at risk for CKD, endstage kidney disease, and cardiovascular disease [6-9].

For a common serious syndrome with dire short- and long-term consequences, progress in diagnosis, prognosis, and therapy has been modest. We need means to diagnose AKI early, foresee the time course of recovery, and predict the risk of progression to CKD. For therapeutics, we need effective prophylaxis of AKI in high-risk situations, accelerate recovery in established AKI, prevent extrarenal complications, and forestall AKI-to-CKD transition. We will explore if FGF23 and aKlotho can fulfill these roles.

\section{FGF23 and AKI}

Multiple factors increase FGF23 production, including high blood pressure, phosphate loading, iron deficiency, hypoxia, and inflammation [10]. FGF23 actions are transduced by the FGF receptor 1 (FGFR1) $[11,12]$ and its coreceptor transmembrane aKlotho [13]. FGF23 can also exert action through binding to FGFR4 in the heart and liver, independently of aKlotho $[14,15]$. Metabolism of FGF23 includes cleavage of bioactive intact FGF23 $(\sim 30 \mathrm{kDa})$ into C-terminal $(\sim 12 \mathrm{kDa})$ and N-terminal $(\sim 18 \mathrm{kDa})$ fragments. FGF23 seems to be also cleared by the kidneys, possibly through filtration and/or catabolism [16]. Circulating FGF23 includes the intact hormone (iFGF23) and the $\mathrm{N}$ - and C-termini. In states such as iron deficiency and inflammation, increased production of FGF23 is matched by FGF23 cleavage, with high levels of FGF23 fragments in circulation [17]. Two assays used to measure circulating FGF23 levels are "Cterminal" (cFGF23) which detects both C-terminal fragments and the full-length peptide and "intact" (iFGF23) which measures only intact FGF23.

Elevated FGF23 levels have been observed in multiple studies of human AKI [10]. Plasma cFGF23 levels were 5.6-fold higher in patients with AKI versus age-matched patients without AKI [18]. Note that increased FGF23 levels detected with the cFGF23 assay (intact + C-term) may reflect increased FGF23 production and/or impaired clearance but do not inform about FGF23 bioactivity. Few studies have used both cFGF23 and iFGF23 assays in conjunction. In adult patients undergoing cardiac surgery, plasma cFGF23 levels were markedly increased $(\sim 100$ fold) postoperatively in patients who did versus did not develop AKI, while iFGF23 levels were modestly ( 2fold) higher postoperatively in AKI versus non-AKI patients [19]. Similar findings were seen in a folic acid ne- phropathy mouse model [20]. Therefore, both production and clearance of FGF23 may be affected in AKI.

In 250 adult patients undergoing cardiac surgery, plasma cFGF23 levels differentially increased at the end of cardiopulmonary bypass in patients with versus without postoperative AKI, predating changes in other mineral metabolites [19]. Performance evaluation of utility of cFGF23 for early AKI diagnosis was modest $(\mathrm{AUC}=0.78)$ but superior to urinary kidney injury biomarkers [19]. Similar observations were derived from cohorts of critically ill patients in which cFGF23 was measured in plasma and urine within $24-48 \mathrm{~h}$ of ICU admission [21, 22].

FGF23 levels may also have prognostic utility in AKI. In a large post hoc analysis ( $N=1,527$ patients), increased risk of 60-day mortality was noted in patients with the highest versus lowest quartiles of cFGF23 ( 3.8-fold) and iFGF23 ( 2.0-fold) [23].

The canonical mineral metabolism regulators of FGF23 production in bones seem not to play a major role in AKI $[20,24]$. Furthermore, contribution of FGF23 production from other organs in acute injury/inflammation settings is not fully understood. FGF23 may augment myofibroblast activation and fibrosis via TGF- $\beta$-related pathways, opening an angle for therapeutic FGF23 blockade $[25,26]$. However, off-target effects of FGF23 such as impairment of immune [27, 28], endothelial [29], and cardiac functions [15] are contenders and require further investigation. Therefore, it is still unclear if anti-FGF23 antibody (burosumab), C-terminal FGF23 (experimentally shown to interfere with FGF23 signaling at supraphysiological concentrations) [30], or specific FGFR4 inhibition have clinical applications in attenuation of incident AKI or its consequences.

\section{aKlotho in AKI}

\section{Diagnosis}

aKlotho deficiency is universal in AKI animal models including ischemia-reperfusion injury (IRI) [31], unilateral ureteric obstruction [32] (UUO), sepsis induced by lipopolysaccharide (LPS) injection or cecal ligation and puncture (CLP) $[33,34]$, and nephrotoxins including cisplatin [35] (CP) and folic acid [36] (FA), indicating that aKlotho downregulation in the kidney is a general phenomenon after acute kidney insults (Table 1). In rats, aKlotho mRNA started to fall $6 \mathrm{~h}$ post-IRI-AKI and returned to baseline around 3-4 days [37]. aKlotho protein in the kidney fell at $3 \mathrm{~h}$ while neutrophil gelatinase-associated lipocalin (NGAL) did not increase until $5 \mathrm{~h}$, so the 


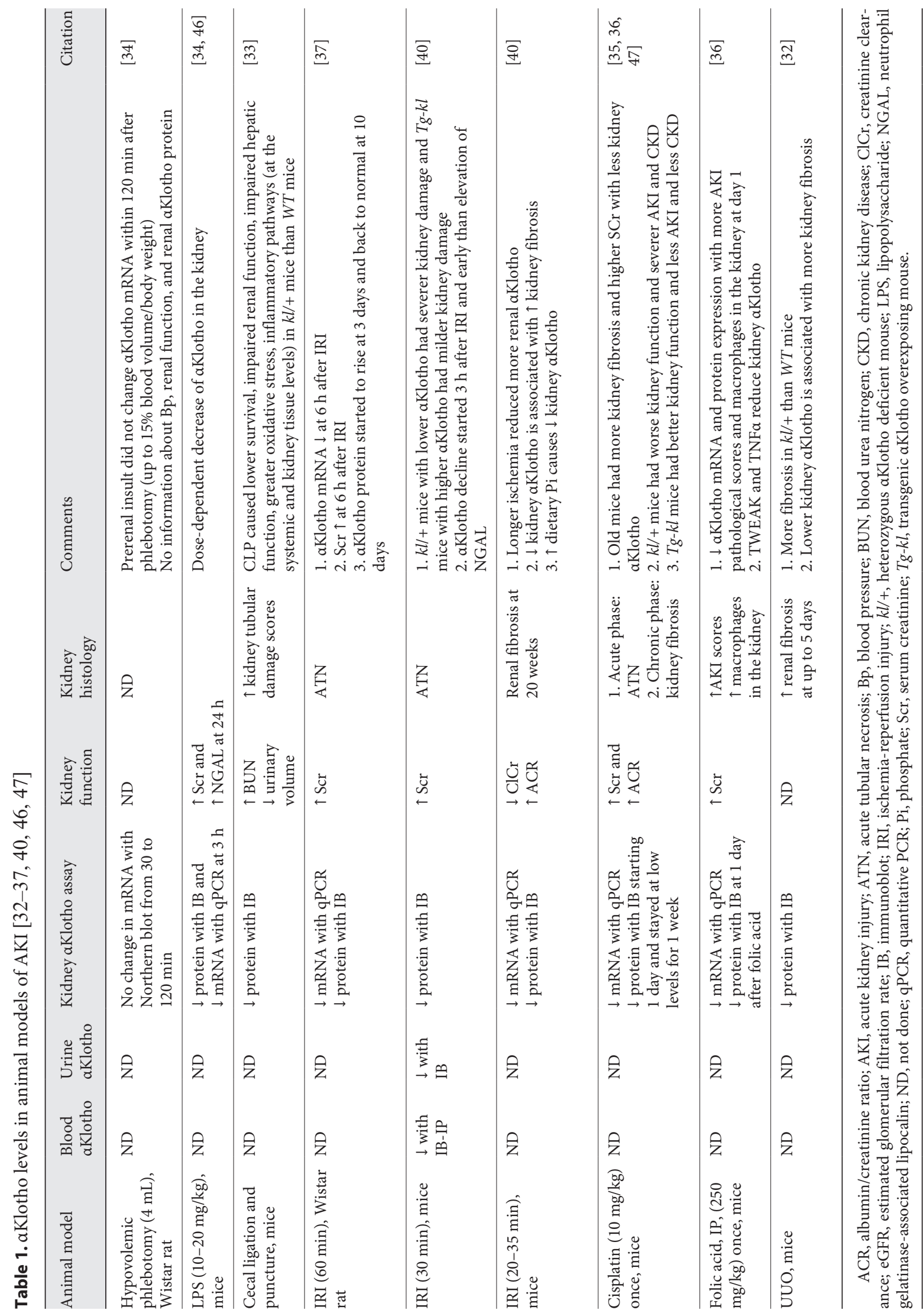




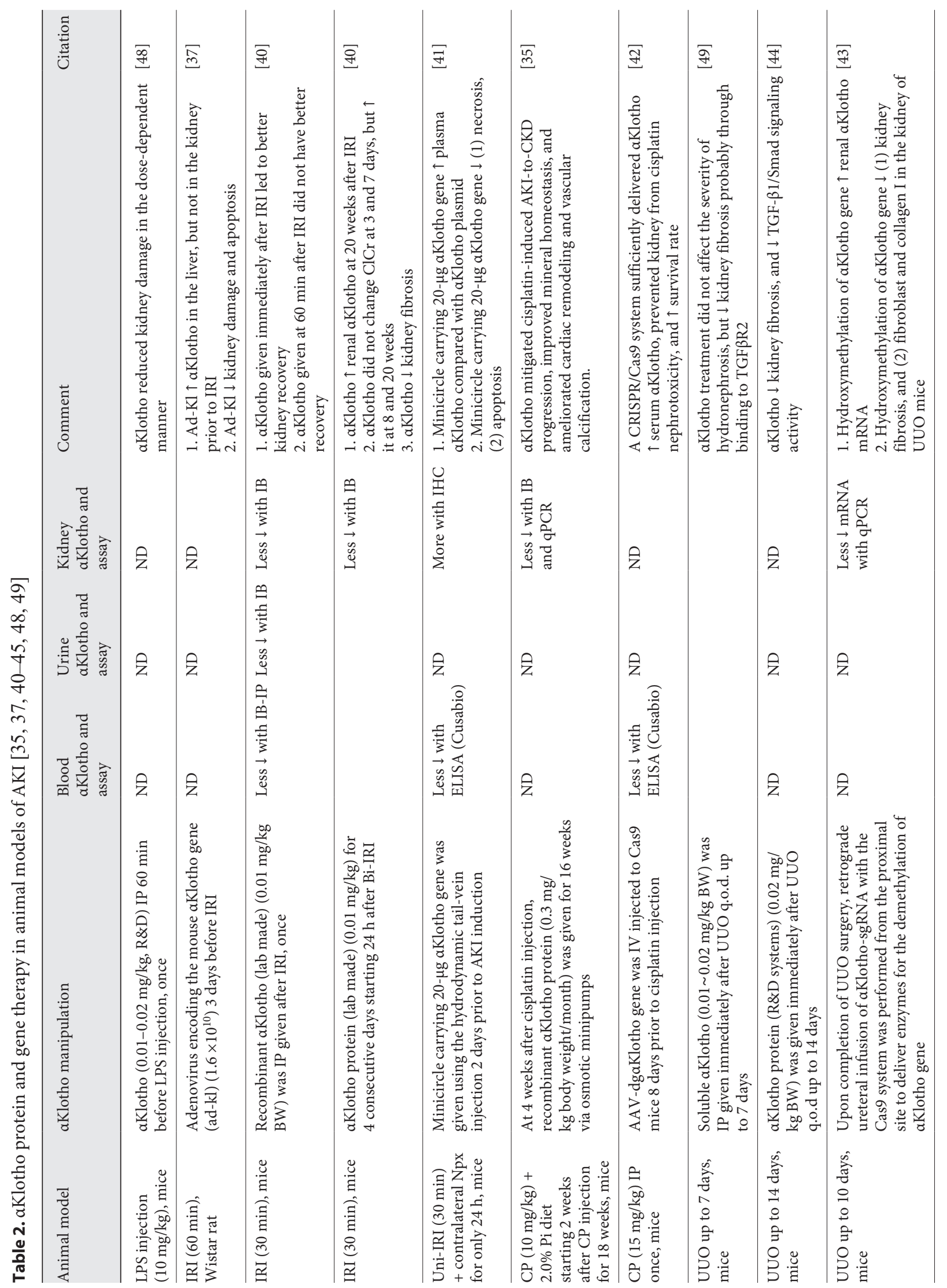




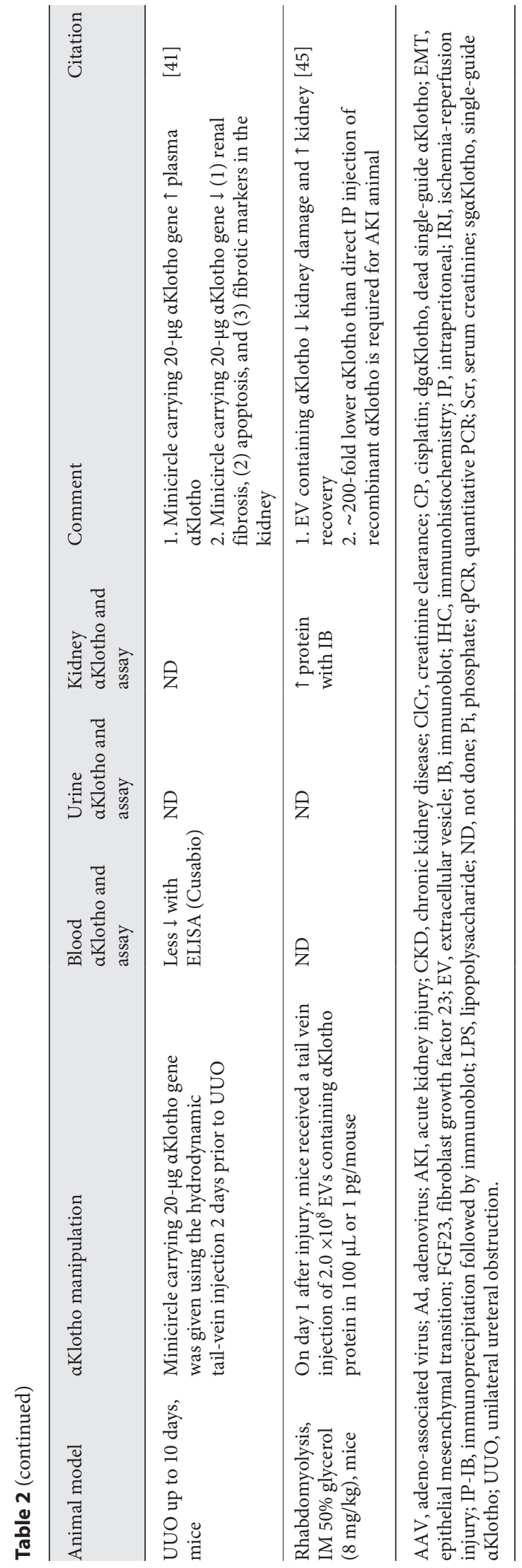

FGF23 and $\alpha$ Klotho in AKI kidney aKlotho protein is an early marker of AKI [38] (Table 1). Urine and plasma aKlotho paralleled changes in kidney aKlotho [38], decreasing dramatically at $3 \mathrm{~h}$, started to recover at $\sim 48 \mathrm{~h}$, and reached normal levels by 7 days. Soluble aKlotho may be a surrogate for kidney aKlotho.

The first human AKI study showed significantly lower urine aKlotho at the time of AKI diagnosis when compared with healthy controls [38]. A larger prospective ICU study showed that AKI patients had lower urinary aKlotho within $48 \mathrm{~h}$ of AKI diagnosis when compared with ICU controls [39]. Urine aKlotho concentration and urine aKlotho-to-Cr ratio inversely correlated with hospital and mechanical ventilation days. Each 1 -fold higher urine $\alpha$ Klotho/Cr was associated with an $83 \%$ lower risk of major adverse kidney events at 90 days [39].

\section{Prognosis}

Heterozygous aKlotho-deficient $(k l /+)$ mice subjected to IRI, CP, sepsis, and UUO had lower aKlothoprotein and more kidney damage than wild-type (WT) mice [34, $35,37,38$ ] (Table 1). Longer ischemia time caused more severe aKlotho deficiency and kidney fibrosis. After recovery of kidney function, lower aKlotho associated with more chronic fibrosis and kidney dysfunction [40], suggesting aKlotho level can predict risk of AKI-to-CKD transition (Table 1).

\section{Prophylaxis}

Mice with genetically high aKlotho before AKI had milder kidney damage and a better renal outcome in IRI [38] and CP-induced AKI [31]. Adenoviral $\alpha$ Klotho gene delivery increased circulating but not kidney aKlotho prior to IRI, ameliorated injury, and kidney dysfunction [37]. aKlotho-bearing minicircle vector increased plasma aKlotho, prevented kidney damage induced by IRI, and attenuated kidney fibrosis in an UUO model [41]. aKlotho overexpression through CRISPR-Cas9 prevented kidney damage and alleviated kidney fibrosis in CP nephrotoxicity [42] and in UUO [43] (Table 2).

\section{Post-AKI Treatment}

Clinical utility mandates efficacy if given after AKI. Recombinant $a$ Klotho protein given to mice immediately after IRI reduced kidney damage [38], but the benefit diminishes dramatically starting $1 \mathrm{~h}$ after the insult. $\alpha$ Klotho administration immediately after UUO followed up to 14 days reduced renal fibrosis, but not hydronephrosis severity [44]. aKlotho-carrying extracellular vesicles [45] promoted kidney recovery in rhabdomyolysis-induced 
Fig. 1. Protracted natural history of AKI and translational opportunities. The various stages of AKI are shown on top corresponding to different levels of kidney function. $\mathrm{Dx}^{1}$, identification of susceptible patients with impending kidney injury; $\mathrm{Dx}^{2}$, early diagnosis of AKI prior to onset of detectable clinical parameters; $\mathrm{Px}^{1}$, prognostic predictor of recovery-phase onset and speed of recovery; $\mathrm{Px}^{2}$, predictor of probability, rapidity, and severity of AKI-toCKD progression; $\mathrm{PPx}^{1}$, prevention of onset of AKI in susceptible patients; $\mathrm{PPx}^{2}$, prevent, delay, or slow down AKI-to-CKD progression. Application in combination with $\mathrm{Px}^{2} ; \mathrm{Rx}^{1}$, promote or accelerate recovery from AKI; $\mathrm{Rx}^{2}$, independent of its effect on kidney function, treatment can be directed to the amelioration of extrarenal complications with cardiovascular complications being a major target. AKI, acute kidney injury; CKD, chronic kidney disease. Dx, diagnosis; Px, prognosis; PPx, prophylaxis; $\mathrm{Rx}$, treatment.

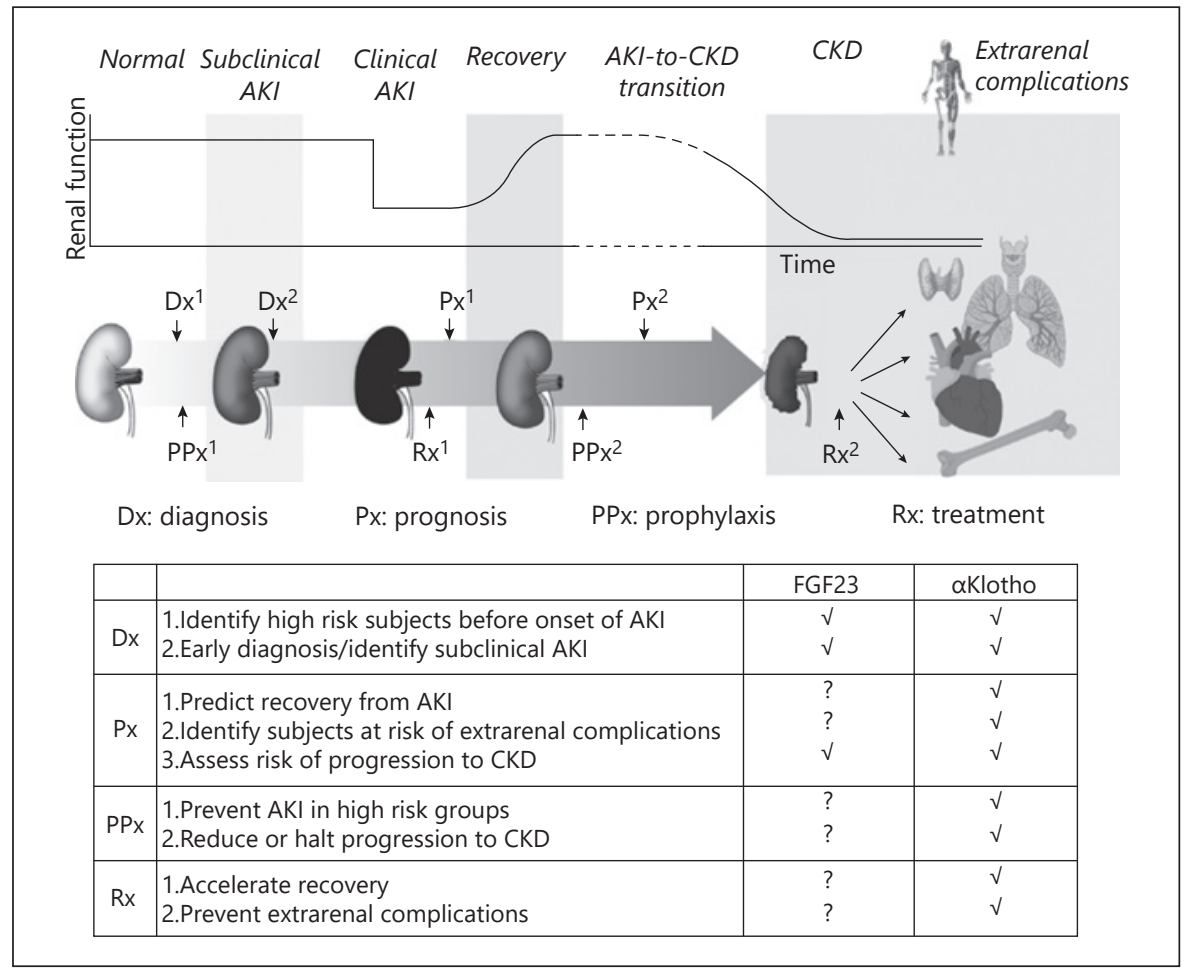

Table 3. Potential modalities for FGF23-aKlotho therapy in AKI

\begin{tabular}{|c|c|c|c|}
\hline \multicolumn{2}{|l|}{ Decrease FGF23 bioactivity } & \multicolumn{2}{|l|}{ Increase aKlotho bioactivity } \\
\hline mechanism & description & mechanism & description \\
\hline Decrease synthesis & Phosphate restriction & Increase endogenous protein & $\begin{array}{l}\text { Phosphate restriction } \\
\text { ACE inhibition } \\
\text { PPAR } \gamma \text { activator } \\
\text { Recombinant } \alpha \text { Klotho itself }\end{array}$ \\
\hline Increase degradation & GALNT3 inhibitor & Integrated transgene & Successful in animals \\
\hline Neutralization & Monoclonal antibody & Gene delivery & Successful in animals \\
\hline Blockade of receptor binding & Experimental development & Recombinant exogenous protein & Successful in animals \\
\hline Blockade of receptor signaling & Experimental development & & \\
\hline
\end{tabular}

FGF23, fibroblast growth factor 23; AKI, acute kidney injury.

AKI. At $24 \mathrm{~h}$ after IRI, when kidney injury was fully established, serum creatinine had peaked, and endogenous aKlotho was at its lowest levels, aKlotho protein injection for 4 consecutive days preserved endogenous kidney aKlotho levels, accelerated recovery, suppressed kidney fibrosis, and protected against AKI-to-CKD transition [40] (Table 2).

\section{Late Treatment}

In 2 CKD models, aKlotho protein was given 4 weeks after CKD induction and sustained for 12 weeks [46], or up to 16 weeks [35]. Plasma aKlotho protein levels, kidney function and fibrosis, and cardiac remodeling/cardiomyopathy were improved possibly via both direct effects and secondary effects due to renoprotection (Table 2).

\section{Summary}

The database of FGF23 is currently composed largely of human studies, which are strong but only associative in nature. In contrast, the aKlotho database is populated by com- 
pelling animal experiments but the clinical data are still in early stages of development. Regarding this pair of proteins with mineral-regulating properties and a plethora of other actions, one can envision many potential applications in human AKI (Fig. 1; Table 3). The potential applications are immense, and further research should be directed at the pathobiology of FGF23 in AKI in preclinical studies to dissect whether it is pathogenic or a biomarker (or both), that is, interventional experiments. Prospective longitudinal clinical studies using simultaneous iFGF23 and cFGF23 assays will enrich our database. Standardization of soluble aKlotho assays will enrich the human database and make it generalizable. The extensive preclinical data in aKlotho are in dire need of human translation in both diagnostics and therapeutics but one faces various hurdles in this effort.

\section{Acknowledgements}

The authors are supported by the National Institutes of Health (R01-DK091392, R01-DK092461, and R01-DK092461-S1 to OWM and $\mathrm{MCH}$ ), the George O'Brien Kidney Research Center (P30-DK-07938 to OWM), the Charles and Jane Pak Center Innovative Research Support (to OWM and $\mathrm{MCH}$ ), and Endowed Professor Collaborative Research Support (to OWM and $\mathrm{MCH}$ ). JAN is a recipient of an Early Career Pilot Grant from the National Center for Advancing Translational Sciences, National Institutes of Health, through Grant UL1TR001998. The authors are grateful to the excellent secretarial assistance provided by Ms. Yesenia Aguirre.

\section{Conflict of Interest Statement}

The authors have no conflicts of interest to disclose.

\section{References}

1 Hu MC, Shiizaki K, Kuro-o M, Moe OW. Fibroblast growth factor 23 and Klotho: physiology and pathophysiology of an endocrine network of mineral metabolism. Annu Rev Physiol. 2013;75:503-33.

2 Edmonston D, Wolf M. FGF23 at the crossroads of phosphate, iron economy and erythropoiesis. Nat Rev Nephrol. 2020;16(1):7-19.

3 Kuro-o M, Matsumura Y, Aizawa H, Kawaguchi H, Suga T, Utsugi T, et al. Mutation of the mouse Klotho gene leads to a syndrome resembling ageing. Nature. 1997;390(6655):4551.

4 Waikar SS, Liu KD, Chertow GM. Diagnosis, epidemiology and outcomes of acute kidney injury. Clin J Am Soc Nephrol. 2008;3(3): 844-61.

5 Silver SA, Long J, Zheng Y, Chertow GM. Cost of acute kidney injury in hospitalized patients. J Hosp Med. 2017;12(2):70-6.

6 Chawla LS, Amdur RL, Amodeo S, Kimmel PL, Palant CE. The severity of acute kidney injury predicts progression to chronic kidney disease. Kidney Int. 2011;79(12):1361-9.

7 Coca SG, Singanamala S, Parikh CR. Chronic kidney disease after acute kidney injury: a systematic review and meta-analysis. Kidney Int. 2012;81(5):442-8.

8 Wald R, Quinn RR, Adhikari NK, Burns KE, Friedrich JO, Garg AX, et al. Risk of chronic dialysis and death following acute kidney injury. Am J Med. 2012;125(6):585-93.

9 Bansal N, Matheny ME, Greevy RA Jr, Eden SK, Perkins AM, Parr SK, et al. Acute kidney injury and risk of incident heart failure among US veterans. Am J Kidney Dis. 2018;71(2): 236-45.

10 Christov M, Neyra JA, Gupta S, Leaf DE. Fibroblast growth factor 23 and klotho in AKI. Semin Nephrol. 2019;39(1):57-75.

11 Yazaki N, Fujita H, Ohta M, Kawasaki T, Itoh $\mathrm{N}$. The structure and expression of the FGF receptor-1 mRNA isoforms in rat tissues. Biochim Biophys Acta. 1993;1172(1-2):3742.

12 Liu S, Vierthaler L, Tang W, Zhou J, Quarles LD. FGFR3 and FGFR4 do not mediate renal effects of FGF23. J Am Soc Nephrol. 2008; 19(12):2342-50.

13 Urakawa I, Yamazaki Y, Shimada T, Iijima K, Hasegawa H, Okawa K, et al. Klotho converts canonical FGF receptor into a specific receptor for FGF23. Nature. 2006;444(7120):7704.

14 Singh S, Grabner A, Yanucil C, Schramm K, Czaya B, Krick S, et al. Fibroblast growth factor 23 directly targets hepatocytes to promote inflammation in chronic kidney disease. Kidney Int. 2016;90(5):985-96.

15 Faul C, Amaral AP, Oskouei B, Hu MC, Sloan A, Isakova T, et al. FGF23 induces left ventricular hypertrophy. J Clin Invest. 2011; 121(11):4393-408.

16 van Ballegooijen AJ, Rhee EP, Elmariah S, de Boer IH, Kestenbaum B. Renal clearance of mineral metabolism biomarkers. J Am Soc Nephrol. 2016;27(2):392-7.

17 Smith ER, Cai MM, McMahon LP, Holt SG. Biological variability of plasma intact and Cterminal FGF23 measurements. J Clin Endocrinol Metab. 2012;97(9):3357-65.

18 Leaf DE, Wolf M, Waikar SS, Chase H, Christov M, Cremers S, et al. FGF-23 levels in patients with AKI and risk of adverse outcomes. Clin J Am Soc Nephrol. 2012;7(8): 1217-23.

19 Leaf DE, Christov M, Jüppner H, Siew E, Ikizler TA, Bian A, et al. Fibroblast growth factor 23 levels are elevated and associated with severe acute kidney injury and death following cardiac surgery. Kidney Int. 2016;89(4):93948.

20 Christov M, Waikar SS, Pereira RC, Havasi A, Leaf DE, Goltzman D, et al. Plasma FGF23 levels increase rapidly after acute kidney injury. Kidney Int. 2013;84(4):776-85.

21 Leaf DE, Jacob KA, Srivastava A, Chen ME, Christov $M$, Jüppner $\mathrm{H}$, et al. Fibroblast growth factor 23 levels associate with $\mathrm{AKI}$ and death in critical illness. J Am Soc Nephrol. 2017;28(6):1877-85.

22 Rygasiewicz K, Hryszko T, Siemiatkowski A, Brzosko S, Rydzewska-Rosolowska A, Naumnik B. C-terminal and intact FGF23 in critical illness and their associations with acute kidney injury and in-hospital mortality. Cytokine. 2018;103:15-9.

23 Leaf DE, Siew ED, Eisenga MF, Singh K, Mc Causland FR, Srivastava A, et al. Fibroblast growth factor 23 associates with death in critically ill patients. Clin J Am Soc Nephrol. 2018;13(4):531-41.

24 Mace ML, Gravesen E, Hofman-Bang J, Olgaard K, Lewin E. Key role of the kidney in the regulation of fibroblast growth factor 23 . Kidney Int. 2015;88(6):1304-13.

25 Smith ER, Holt SG, Hewitson TD. FGF23 activates injury-primed renal fibroblasts via FGFR4-dependent signalling and enhancement of TGF- $\beta$ autoinduction. Int J Biochem Cell Biol. 2017;92:63-78.

26 Smith ER, Tan SJ, Holt SG, Hewitson TD. FGF23 is synthesised locally by renal tubules and activates injury-primed fibroblasts. Sci Rep. 2017;7(1):3345

27 Bacchetta J, Sea JL, Chun RF, Lisse TS, Wesseling-Perry K, Gales B, et al. Fibroblast growth factor 23 inhibits extrarenal synthesis of 1,25-dihydroxyvitamin $\mathrm{D}$ in human monocytes. J Bone Miner Res. 2013;28(1): 46-55.

28 Rossaint J, Oehmichen J, Van Aken H, Reuter S, Pavenstädt HJ, Meersch M, et al. FGF23 signaling impairs neutrophil recruitment and host defense during CKD. J Clin Invest. 2016; 126(3):962-74 
29 Silswal N, Touchberry CD, Daniel DR, McCarthy DL, Zhang S, Andresen J, et al. FGF23 directly impairs endothelium-dependent vasorelaxation by increasing superoxide levels and reducing nitric oxide bioavailability. Am J Physiol Endocrinol Metab. 2014;307(5): E426-36.

30 Goetz R, Nakada Y, Hu MC, Kurosu H, Wang L, Nakatani T, et al. Isolated C-terminal tail of FGF23 alleviates hypophosphatemia by inhibiting FGF23-FGFR-Klotho complex formation. Proc Natl Acad Sci U S A. 2010; 107(1):407-12.

31 Panesso MC, Shi M, Cho HJ, Paek J, Ye J, Moe $\mathrm{OW}$, et al. Klotho has dual protective effects on cisplatin-induced acute kidney injury. Kidney Int. 2014;85(4):855-70.

32 Sugiura H, Yoshida T, Shiohira S, Kohei J, Mitobe M, Kurosu H, et al. Reduced Klotho expression level in kidney aggravates renal interstitial fibrosis. Am J Physiol Renal Physiol. 2012;302(10):F1252-64.

33 Jorge LB, Coelho FO, Sanches TR, Malheiros DMAC, Ezaquiel de Souza L, Dos Santos F, et al. Klotho deficiency aggravates sepsis-related multiple organ dysfunction. Am J Physiol Renal Physiol. 2019;316(3):F438-48.

34 Ohyama Y, Kurabayashi M, Masuda H, Nakamura T, Aihara Y, Kaname T, et al. Molecular cloning of rat klotho cDNA: markedly decreased expression of klotho by acute inflammatory stress. Biochem Biophys Res Commun. 1998;251(3):920-5.

35 Shi M, McMillan KL, Wu J, Gillings N, Flores $\mathrm{B}$, Moe OW, et al. Cisplatin nephrotoxicity as a model of chronic kidney disease. Lab Invest. 2018;98(8):1105-21.
36 Moreno JA, Izquierdo MC, Sanchez-Niño MD, Suárez-Alvarez B, Lopez-Larrea C, Jakubowski A, et al. The inflammatory cytokines TWEAK and TNFa reduce renal klotho expression through NFkB. J Am Soc Nephrol. 2011;22(7):1315-25.

37 Sugiura H, Yoshida T, Tsuchiya K, Mitobe M, Nishimura S, Shirota S, et al. Klotho reduces apoptosis in experimental ischaemic acute renal failure. Nephrol Dial Transplant. 2005; 20(12):2636-45.

38 Hu MC, Shi M, Zhang J, Quiñones H, Kuro-o M, Moe OW. Klotho deficiency is an early biomarker of renal ischemia-reperfusion injury and its replacement is protective. Kidney Int. 2010;78(12):1240-51.

39 Neyra JA, Li X, Mescia F, Ortiz-Soriano V, Adams-Huet B, Pastor J, et al. Urine klotho is lower in critically ill patients with versus without acute kidney injury and associates with major adverse kidney events. Crit Care Explor. 2019;1(6):e0016.

40 Shi M, Flores B, Gillings N, Bian A, Cho HJ, Yan S, et al. aKlotho mitigates progression of AKI to CKD through activation of autophagy. J Am Soc Nephrol. 2016;27(8):2331-45.

41 Shin YJ, Luo K, Quan Y, Ko EJ, Chung BH, Lim SW, et al. Therapeutic challenge of minicircle vector encoding klotho in animal model. Am J Nephrol. 2019;49(5):413-24.

42 Liao HK, Hatanaka F, Araoka T, Reddy P, Wu $\mathrm{MZ}$, Sui $\mathrm{Y}$, et al. In vivo target gene activation via CRISPR/Cas9-mediated trans-epigenetic modulation. Cell. 2017;171(7):1495-1507. e15.

43 Xu X, Tan X, Tampe B, Wilhelmi T, Hulshoff MS, Saito S, et al. High-fidelity CRISPR/Cas9based gene-specific hydroxymethylation rescues gene expression and attenuates renal fibrosis. Nat Commun. 2018;9(1):3509.
44 Li S, Yu L, He A, Liu Q. Klotho inhibits unilateral ureteral obstruction-induced endothelial-to-mesenchymal transition. Front Pharmacol. 2019;10:348.

45 Grange C, Papadimitriou E, Dimuccio V, Pastorino C, Molina J, O'Kelly R, et al. Urinary extracellular vesicles carrying klotho improve the recovery of renal function in an acute tubular injury model. Mol Ther. 2020;28(2): 490-502.

$46 \mathrm{Hu}$ MC, Shi M, Gillings N, Flores B, Takahashi M, Kuro OM, et al. Recombinant alphaKlotho may be prophylactic and therapeutic for acute to chronic kidney disease progression and uremic cardiomyopathy. Kidney Int. 2017;91(5):1104-14.

47 Ueno T, Kobayashi N, Nakayama M, Takashima Y, Ohse T, Pastan I, et al. Aberrant Notch1-dependent effects on glomerular parietal epithelial cells promotes collapsing focal segmental glomerulosclerosis with progressive podocyte loss. Kidney Int. 2013;83(6): 1065-75.

48 Liu X, Niu Y, Zhang X, Zhang Y, Yu Y, Huang $\mathrm{J}$, et al. Recombinant alpha-klotho protein alleviated acute cardiorenal injury in a mouse model of lipopolysaccharide-induced septic cardiorenal syndrome type 5. Anal Cell Pathol. 2019;2019:5853426.

49 Doi S, Zou Y, Togao O, Pastor JV, John GB, Wang L, et al. Klotho inhibits transforming growth factor-beta1 (TGF-beta1) signaling and suppresses renal fibrosis and cancer metastasis in mice. J Biol Chem. 2011;286(10): 8655-65. 